\title{
Hemodynamic and Cardiovascular Disorders During Surrenalectomy for Pheochromocytoma
}

\section{Taoufik Elabbassi*, Mohamed Ouchane, Issam Hamrerras and Mohamed Rachid Lefriyekh}

Department of General Surgery, University Hospital Center Ibn Rochd, Faculty of

Medicine and Pharmacy, Hassan II University, Casablanca, Morocco

*Corresponding Author: Taoufik Elabbassi, Department of General Surgery,

University Hospital Center Ibn Rochd, Faculty of Medicine and Pharmacy, Hassan

II University, Casablanca, Morocco.
Received: February 19, 2020

Published: March 04, 2020

(C) All rights are reserved by Taoufik

Elabbassi., et al.

\section{Abstract}

The pheochromocytoma is responsible for the excessive release of adrenaline and norepinephrine which leads to hypertensive flare-ups, dysrhythmias, strokes and pre-operative myocardial ischemia. This makes perioperative hemodynamic instability the greatest surgical and anesthetic challenge in surgery for a pheochromocytoma, hence the need for patient preparation with appropriate medications. This hemodynamic instability occurs in a large proportion of post-operative patients who often require vasopressor agents. Management of these hemodynamic disorders has improved outcomes by reducing both mortality and morbidity.

We report a case of a pheochromocytoma patient who underwent adrenalectomy, whose cardiac problems were aggravated postoperatively and were controlled by the administration of vasoactive drugs in the resuscitation setting.

Keywords: Pheochromocytoma; Adrenalectomy; Surgery; Cardiovascular Complications

\section{Introduction}

Pheochromocytoma is a rare neuroendocrine tumour, developed at the expense of the chromaffin cells of the adrenal glands, which secretes in excess the catecholamines responsible for the major hemodynamic changes with cardiovascular complications [1]. The curative treatment of pheochromocytoma is based on surgery but requires preoperative preparation and conditioning of the blood pressure and specific postoperative monitoring [2].

We report the case of a patient operated on for an enormous left adrenal pheochromocytoma, who had benefited from a left adrenalectomy whose immediate postoperative consequences were marked by the occurrence of severe left heart failure.

Patient and observation

This is a 42-year-old patient with no particular pathological history who had been presenting for several months with heavy- type left hypochondrial pain associated with dizziness, headache, sweating and palpitations, evolving in a context of apyrexia and preservation of general condition, The clinical examination found a conscious patient with respiratory stability, hypertension at 13/06 mmhg, positive left lumbar contact without hepatomegaly or splenomegaly, pelvic touches were normal, cardiovascular examination showed a peak shock in place, sounds B1 and B1 well perceived, absence of heart murmur. A holter-ECG detected voltage peaks, maximum at 164/80 mmhg and minimum at 114/65 mmh, heart rate varied between 92 and 72 beats per minute (Table 1). The preoperative ECG showed no electrical abnormalities, and the cardiac ultrasound was normal with a $65 \%$ ejection fraction with no associated valvulopathy. The abdominal CT scan showed a voluminous solidocystics process of the left hypochondrium measuring $12 \mathrm{~cm}$ long axis, the methoxylated derivatives of the catecholamines were very positive, Normetanephrine > 4050 nmol/24h, Metadrenaline $>3870 \mathrm{nmol} / 24 \mathrm{~h}$, all were in favour of a left pheochromocytoma. 


\begin{tabular}{|l|c|c|c|c|}
\hline & Maximal & Minimal & Mean & $\begin{array}{c}\text { Sdt } \\
\text { Dev }\end{array}$ \\
\hline Systolic (mmHg) & 164 & 114 & 132.7 & 22.3 \\
\hline Diastolic (mmHg) & 80 & 65 & 71.3 & 6.4 \\
\hline heart's rate (per minute) & 92 & 79 & 80 & 8.6 \\
\hline MAP (mmHg) & 116 & 76 & 92 & \\
\hline
\end{tabular}

Table 1 : 24 hour blood pressure monitoring showing blood pressure oscillations.

The patient was operated on by median laparotomy with, on surgical exploration, the presence of a $15 \mathrm{~cm}$ diameter solidocystic left adrenal mass without locoregional extension, a left adrenalectomy was performed (Figure 1).

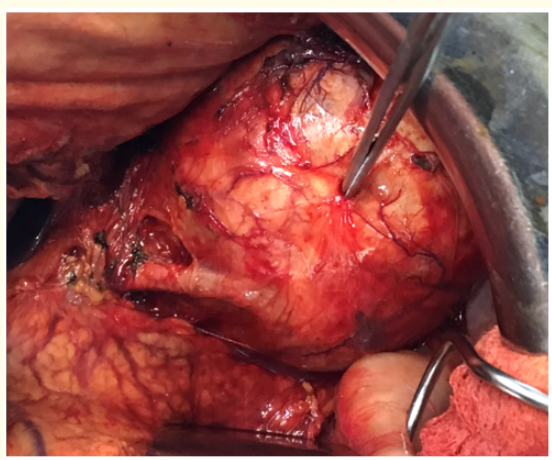

Figure 1: Intraoperative image of the pheochromocytoma (the arrow).

Intraoperatively, no hemodynamic instability at mobilization and resection of the tumor was marked, however, in the immediate postoperative period, hypotension with tachycardia at 149 beats per minute was noted, the control cardiac ultrasound showed left heart failure with a ventricular ejection fraction of $15 \%$ and the presence of a pericardial effusion blade facing the right cavities. An ECG had shown the presence of repolarization disorders related to tachycardia (Figure 2).

The patient was placed on dobutamine $10 \mu \mathrm{g} / \mathrm{kg} / \mathrm{min}$ in an electric syringe, norepinephrine $3 \mu \mathrm{g} / \mathrm{kg} / \mathrm{min}$ and furosemide $20 \mathrm{mg}$ for 2 days in a surgical resuscitation setting. Cardiac function improved with a control left ventricular ejection fraction of $55 \%$. She was controlled after 6 months of follow-up, her blood pressure figures were normal and her clinical sequelae were unremarkable.

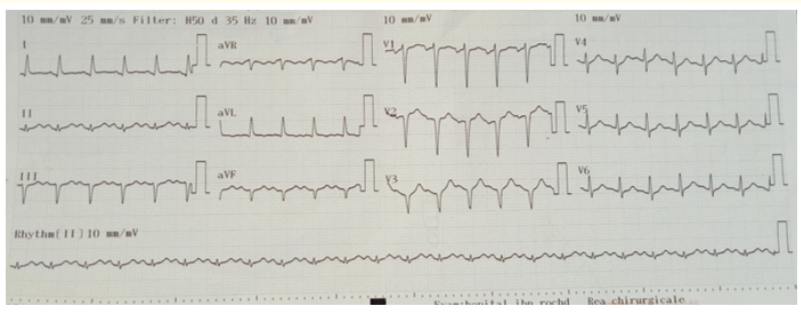

Figure 2: ECG showing sinus tachycardia with repolarization disorder.

\section{Discussion}

Pheochromocytomas are rare neuroendocrine tumours that develop at the expense of the adrenal medulla responsible for an excess secretion of catecholamines, causing high blood pressure and possible acute cardiovascular complications [3], Clinical symptoms are explained by the release of norepinephrine responsible for vasoconstriction and hypertension and adrenaline responsible for vasodilatation, flush syndrome and postural hypotension, both hormones accelerate the heart rate [4]. The pheochromocytomas secreting dopamine are rarer, responsible for specific signs such as abdominal pain and asthenia [5], the massive release of catecholamines can induce a sudden myocardial sideration responsible for left or global heart failure, or sometimes a true state of cardiogenic shock [3], typical clinical signs associate paroxysmal hypertension with headache, palpitations, profuse sweating, pallor, abdominal or chest pain, visual disturbances are present in only one third of cases [4]. Surgical excision is the only curative treatment for pheochromocytoma. The difficulty and risks of this surgery take into account the surgical procedure and especially the perioperative management and anaesthesia, which requires a multidisciplinary team [2], the dissection of the tumour will lead to the release of catecholamines responsible for hypertensive flare-ups, dysrhythmias, enemas and strokes, or myocardial ischemia [6]. These surgical disturbances can be controlled by the intraoperative use of drugs such as alpha-blockers, beta-blockers, calcium channel blockers including nicardipine, nitroprusside, nitro-glycerine, magnesium and especially magnesium sulfate [1]. After tumour removal, a drop in circulating catecholamine levels can lead to profound hypotension and sometimes death from cardiogenic shock and therefore the anaesthetist must be alerted and cooperate fully with the surgeon $[7,9]$. In postoperative hypotension following adrenalectomy must be controlled in the hours following surgery, it can persist despite 
the administration of a vasopressor and can go on for more than 24 to 48 hours in $40 \%$ of cases [8]. In our case, in addition to postoperative hypotension, the patient presented left heart failure with an ejection fraction of $15 \%$ requiring the administration of dobutamine and noradrenaline until the improvement of his cardiac function.

\section{Conclusion}

The pheochromocytoma is responsible for hemodynamic disorders following the excessive secretion of catecholamines, which leads to alterations in the myocardium that can be aggravated intraoperatively or even postoperatively. Multidisciplinary perioperative management is essential to limit morbidity and even mortality in this type of endocrine pathology.

\section{Consent}

As per international standard or university standard, patient's consent has been collected and preserved by the authors.

\section{Ethical Approval}

As per international standard, written ethical approval has been collected and preserved by all the authors ten days before the submission.

\section{Competing Interests}

All the Authors have declared that no competing interests exist.

\section{Bibliography}

1. Magagi Amadou., et al. "Particularity of anesthesia for surgical treatment of pheochromocytoma: about a case". Pan African Medical Journal 29.1 (2018): 1-4.

2. Grise P and Kuhn J. "Surgical treatment of pheochromocytoma”. Nephrology-Urology (2002): 18-185.

3. Loosli Nicolas., et al. "Pheochromocytoma and paraganglioma”. Swiss Medical Journal 10.441 (2014): 1650-1652.

4. Pacak Karel. "Preoperative management of the pheochromocytoma patient". The Journal of Clinical Endocrinology and Metabolism 92.11 (2007): 4069-4079.

5. Vandwalle J., et al. "Pheochromocytoma and heart failure: an exceptional indication for emergency or semi-emergency adrenalectomy". Advances in Urology 20.7 (2010): 498-502.

6. Thompson Jesse E., et al. "Pheochromocytoma; surgical and anesthetic management". Anesthesiology 15.6 (1954): 658665.
7. Pisarska Magdalena., et al. "Perioperative hemodynamic instability in patients undergoing laparoscopic adrenalectomy for pheochromocytoma". Gland Surgery 5.5 (2016): 506.

8. Desmonts JM and Et Marty JI. "Anaesthetic management of patients with phaeochromocytoma". British Journal of Anaesthesia 56.7 (1984): 781-789.

9. Akiba Masako., et al. "Hypoglycemia induced by excessive rebound secretion of insulin after removal of pheochromocytoma”. World Journal of Surgery 14.3 (1990): 317-324.

\section{Assets from publication with us}

- Prompt Acknowledgement after receiving the article

- Thorough Double blinded peer review

- Rapid Publication

- Issue of Publication Certificate

- High visibility of your Published work

Website: https://www.actascientific.com/

Submit Article: https://www.actascientific.com/submission.php

Email us: editor@actascientific.com

Contact us: +919182824667 